ISSN 0103-5150

Fisioter. Mov., Curitiba, v. 25, n. 2, p. 343-349, abr./jun. 2012

Licenciado sob uma Licença Creative Commons

\title{
Efeito de um programa de exercícios direcionados à mobilidade torácica na DPOC
}

\author{
The effect of an exercise program directed \\ to the thoracic mobility in COPD
}

\author{
Claudiane Pedro Rodrigues ${ }^{[a]}$, Luiz Antonio Alves ${ }^{[b]}$, Tiemi Matsuo ${ }^{[c]}$, \\ Cristiane Golias Gonçalves ${ }^{[\mathrm{d}]}$, Daniela Hayashi ${ }^{[\mathrm{e}]}$
}

[a] Fisioterapeuta especialista em Fisioterapia Respiratória, docente e supervisora de estágio na disciplina de Cardiorrespiratória na Faculdade de Apucarana, Apucarana, PR - Brasil, e-mail: claufisio@yahoo.com.br

[b] Fisioterapeuta especialista em Fisioterapia Respiratória, Mestre em Medicina e Ciências da Saúde, docente e supervisor de estágio na disciplina de Cardiorrespiratória no Centro Universitário Filadélfia (UNIFIL), Londrina, PR - Brasil.

[c] Doutora em Estatística e Experimentação Agronômica pela Escola Superior de Agricultura Luiz de Queiroz, professora associada da Universidade Estadual de Londrina (UEL), Londrina, PR - Brasil.

[d] Fisioterapeuta especialista em Fisioterapia Respiratória, docente e supervisora de estágio na Disciplina Hospitalar na Faculdade de Apucarana, Apucarana, PR - Brasil.

[e] Especialista em Fisioterapia Respiratória, docente na área de Fisioterapia Respiratória Hospitalar na Faculdade de Apucarana (FAP), Apucarana, PR - Brasil.

\section{Resumo}

Introdução: A Doença Pulmonar Obstrutiva Crônica produz alterações mecânicas na caixa torácica que desencadeiam disfunções físicas limitantes das atividades de vida diária e da qualidade de vida. Objetivos: Avaliar os efeitos de um programa de exercícios para readequação do complexo toracopulmonar na mobilidade da caixa torácica, capacidade de exercício e qualidade de vida em pacientes com DPOC. Materiais e métodos: Este estudo avaliou 13 pacientes com DPOC por meio de Espirometria, Teste da Caminhada dos Seis Minutos (TC6min), Cirtometria e Questionário de Qualidade de Vida Saint George (SGRQ). O programa foi elaborado com base em exercícios que objetivam o aumento da mobilidade de caixa torácica, tolerância ao exercício e melhora na resposta subjetiva em relação à qualidade de vida. Resultados: Após 12 semanas de tratamento, verificou-se um aumento significativo na mobilidade da região inferior da caixa torácica (expansibilidade da região xifoide: de $3 \pm 2 \mathrm{~cm}$ para $7 \pm 4 \mathrm{~cm}, \mathrm{p}=0,01$ ), na região abdominal (expansibilidade 
umbilical: de $2 \pm 1 \mathrm{~cm}$ para $6 \pm 4 \mathrm{~cm}, \mathrm{p}=0,01)$ e melhora na distância percorrida no TC6min $(391 \pm 117 \mathrm{~m}$ inicial para final de $442 \pm 124 \mathrm{~m}, \mathrm{p}=0,04$ ). Na avaliação pelo SGRQ, houve uma tendência sem significância estatística de melhora nos três domínios (sintomas: $37 \pm 22$ para $26 \pm 21$; atividades: $64 \pm 15$ para $62 \pm$ 19; e impacto: $42 \pm 16$ para $38 \pm 16$ ). Conclusão: 0 programa de exercícios respiratórios direcionados ao aumento da mobilidade da caixa torácica melhorou a expansibilidade torácica e abdominal e a capacidade de exercício.

Palavras-chave: Mobilidade de caixa torácica. Fisioterapia. DPOC.

\section{Abstract}

Background: Chronic Obstructive Pulmonary Disease produces mechanical changes in chest wall and this physical disability reduces the daily life activities and quality of life levels. Objectives: To assess the effects of an exercise program for Thoracopulmonary Complex Re-Adaptation in the chest wall mobility, exercise capacity and quality of life in COPD patients. Materials and methods: Thirteen COPD patients were studied. Spirometry, Six Minutes Walk Test, Thoracic Mobility and Quality of Life by Saint George Respiratory Questionnaire (SGRQ) were the variables. The exercise program was created based on physical exercises that aim at increasing the thoracic mobility and a better exercise tolerance, as well as improving the subjective response related to quality of life. Results: After a 12-week program, it was observed a significant improvement in chest wall mobility in lower chest region (xiphoid level mobility: from $3 \pm 2 \mathrm{~cm}$ to $7 \pm 4 \mathrm{~cm} ; p=0.01$ ) and in the abdominal region (umbilical level mobility: from $2 \pm 1 \mathrm{~cm}$ to $6 \pm 4 \mathrm{~cm} ; p=0.01$ ). There was a statistically significant improvement of exercise capacity (walk distance: from $391 \pm 117 \mathrm{~m}$ to $442 \pm 124 \mathrm{~m} ; p=0.04$ ). In quality of life assessment by SGRQ, there was a tendency of improving in three domains (symptom: from $37 \pm 22$ to $26 \pm 21$; activity: from $64 \pm 15$ to $62 \pm 19$; and impacts: from $42 \pm 16$ to $38 \pm 16$ ), but with no statistic significances. Conclusion: Respiratory exercises aimed at increasing the chest wall mobility improve abdominal and lower chest expansibility and exercise tolerance.

Keywords: Chest wall mobility. Physical therapy. COPD.

\section{Introdução}

A Doença Pulmonar Obstrutiva Crônica (DPOC) tem como característica principal uma alteração da função pulmonar associada à disfunção dos músculos esqueléticos periféricos, acarretando intolerância ao exercício e piora progressiva do condicionamento físico, com consequente isolamento social, ansiedade, depressão e dependência (1-6), e geração de um ciclo vicioso com comprometimento da qualidade de vida desses indivíduos (7).

Os músculos respiratórios fazem parte do complexo toracopulmonar e atuam na respiração e na estabilidade do tronco $(8,9)$. Na DPOC, a mecânica respiratória é prejudicada em virtude da hiperinsuflação pulmonar, alterando a geometria da parede torácica e reduzindo a zona de aposição do diafragma, alterando a sua ação (10-13).

A fisioterapia respiratória busca interferir nesses mecanismos, melhorando a capacidade funcional do doente e restituindo sua independência. Para tanto, dispõe de condutas variadas, como Manobras de Higiene Brônquica, Recondicionamento Físico e Treinamento dos Músculos Respiratórios (14). Além disso, a Cinesioterapia Respiratória dispõe de exercícios específicos que visam à Readequação do Complexo Toracopulmonar (RCTP), empregando exercícios ativos de tronco, membros superiores e inferiores associados à reeducação diafragmática. Ocorre um aumento da eficiência dos músculos respiratórios e um auxílio no mecanismo fisiológico da bomba ventilatória, uma vez que a obstrução crônica das vias aéreas leva a um comprometimento progressivo da eficiência e perda da capacidade funcional (15).

A Cinesioterapia Respiratória não está descrita mundialmente como um componente de programas de Reabilitação Pulmonar, sendo raros os estudos que investigam os benefícios desses exercícios na condição funcional do paciente com DPOC $(10,15)$, mas é aplicada frequentemente na prática clínica. 
Em função dos argumentos acima citados, o objetivo deste estudo foi avaliar o efeito do protocolo de exercícios de RCTP na mobilidade da caixa torácica, na capacidade de exercício e na qualidade de vida dos indivíduos com DPOC.

\section{Casuística e métodos}

A partir de uma amostragem de conveniência, foram estudados 11 pacientes (nove do sexo feminino) com diagnóstico médico de DPOC, gravidade pela Espirometria variando de Leve a Grave, e quadro clínico estável há pelo menos três meses. Nenhum paciente tinha diagnóstico de doença cardíaca, quadro exacerbado da doença pulmonar, dificuldade em deambular e deficit cognitivo (Tabela 1).

Os pacientes foram encaminhados ao Ambulatório de Fisioterapia Cardiorrespiratória na Clínica Escola da FAP (Faculdade de Apucarana, Apucarana/PR) por meio de solicitação médica. Após tomarem conhecimento deste estudo, aceitaram participar e assinaram o Termo de Consentimento Livre e Esclarecido aprovado pelo Comitê de Ética em Pesquisas Envolvendo Seres Humanos (CETi-FAP, parecer n. 040/007).

Os pacientes foram submetidos a uma avaliação inicial e final após as sessões de RCTP, constituída por:

1) Espirometria: Realizada com aparelho portátil da marca VITALOGRAPH ${ }^{\circledR}$ MICRO (Vitalograph Ltd - Irlanda) segundo as normas descritas pelo II Consenso Brasileiro sobre Espirometria (16).

2) Cirtometria Torácica: Realizada para mensurar a expansibilidade torácica, utilizando uma

Tabela 1 - Caracterização da amostra estudada

\begin{tabular}{lc}
\hline Variável & Valor (média $\pm \mathrm{dpm}$ ) \\
\hline Sexo & $9 \mathrm{M} / 2 \mathrm{H}$ \\
Idade (anos) & $63 \pm 12$ \\
IMC $\left(\mathrm{kg} / \mathrm{m}^{2}\right)$ & $29 \pm 5$ \\
CVF $(\mathrm{em} \mathrm{L} \mathrm{e} \mathrm{\% )}$ & $2,15 \pm 1,30(66 \% \pm 26 \%)$ \\
VEF $_{1}(\mathrm{em} \mathrm{L} \mathrm{e} \mathrm{\% )}$ & $1,28 \pm 0,59(58 \% \pm 21 \%)$ \\
\hline
\end{tabular}

Fonte: Dados da pesquisa.

Legenda: IMC = Índice de Massa Corpórea; CVF = Capacidade Vital Forçada; $\mathrm{VEF}_{1}=$ Volume Expiratório Forçado no 10 segundo. fita métrica posicionada sobre a caixa torácica do paciente nas regiões axilar, xifoide e umbilical. Cada medida foi obtida após solicitar ao individuo uma expiração máxima, seguida de uma inspiração máxima e outra expiração máxima. As medidas foram repetidas duas vezes, sendo registrados os maiores valores e a diferença entre as medidas de inspiração e expiração máximas.

3) Teste da Caminhada dos Seis Minutos: Foi realizado de acordo com as diretrizes da ATS, utilizando um corredor plano, sem acompanhamento e com incentivo verbal (17). Os parâmetros de Pressão Arterial (PA), Frequência Cardíaca (FC), Saturação de Oxigênio $\left(\mathrm{SpO}_{2}\right)$ e o Índice de Percepção Esforço de Borg (IPB) foram verificados no início e ao fim do teste.

4) Qualidade de Vida: Foi utilizado o Questionário Saint George na Doença Respiratória (SGRQ), dividido em três domínios: sintomas, atividade e impacto (18).

0 programa de exercícios foi realizado duas vezes por semana, com duração de 60 minutos cada sessão. Os sinais vitais (PA, FC, FR) e ausculta pulmonar foram verificados no início e ao final de cada sessão. Caso houvesse necessidade de higiene brônquica, o paciente realizava inaloterapia conforme prescrição médica e manobras de higiene brônquica.

Foi utilizado um protocolo de exercícios de RCTP para pacientes com DPOC (15). Eles eram orientados a realizar um padrão respiratório diafragmático ao iniciar o movimento e expirar o ar com os lábios semicerrados (freno labial) durante a execução do movimento $(19,20)$. A sequência das posturas utilizadas segue a evolução do desenvolvimento motor humano, iniciando os exercícios em decúbito dorsal, seguido por sedestação, quadripedia de joelhos e, finalmente, em bipedestação.

Foram realizadas 15 repetições de cada exercício, sendo previamente ensinados de forma demonstrativa aos pacientes. Caso o paciente apresentasse algum desconforto durante a execução dos exercícios, ele era orientado a sentar-se e retornar assim que possível para cumprir as séries, ou fazer uso de oxigenoterapia durante a realização dos exercícios. 0 programa original é constituído de quatro séries progressivas, cada uma com duração de cerca de um mês de tratamento, porém no nosso estudo foram realizadas apenas as séries I, II e III, pelo fato de o questionário 
de qualidade de vida adotado ser validado para avaliações apenas após três meses de tratamento. A duração do programa foi de 12 semanas, constituindo um total de 24 sessões.

A análise estatística foi efetuada no programa SPSS 13.0. As variáveis foram descritas em média \pm desvio padrão médio (dpm). A evolução das medidas, mensurada pela diferença entre os valores finais e iniciais, foi avaliada pelo teste de t para amostras pareadas nos casos em que a variável apresentou distribuição gaussiana pelo teste de Shapiro-Wilk e homogeneidade de variâncias pelo teste de Bartlett; em caso contrário, empregou-se o Wilcoxon para amostras pareadas. Os testes são bicaudais e o nível de significância adotado foi de $5 \%$.

\section{Resultados}

Na Tabela 2 estão apresentados os valores iniciais e finais da Espirometria, domínios do SGRQ, do TC6min e da Cirtometria Torácica. Houve diferença

Tabela 2 - Valores iniciais e finais da Espirometria, Qualidade de Vida, distância percorrida no TC6min e Cirtometria Torácica

\begin{tabular}{lccc}
\hline & Inicial & Final & $\mathbf{p}$ \\
\hline CVF (em L e \%) & $2,15 \pm 1,30$ & $1,81 \pm 0,89$ & $0,2966^{*}$ \\
& $(66 \% \pm 26 \%)$ & $(60 \% \pm 25 \%)$ & \\
VEF $_{1}$ (em L e \%) & $1,28 \pm 0,59$ & $1,28 \pm 0,67$ & $0,9870^{*}$ \\
& $(58 \% \pm 21 \%)$ & $(54 \% \pm 19 \%)$ & \\
Sintomas (SGRQ) & $37 \pm 22$ & $26 \pm 21$ & $0,1307^{\dagger}$ \\
Atividade (SGRQ) & $64 \pm 15$ & $62 \pm 19$ & $0,6248^{\dagger}$ \\
Impacto (SGRQ) & $42 \pm 16$ & $38 \pm 16$ & $0,3505^{\dagger}$ \\
TC6min (m) & $391 \pm 117$ & $442 \pm 124$ & $0,0403^{*}$ \\
Cirtometria & & & \\
$\quad$ Ápice & $5 \pm 3$ & $8 \pm 4$ & $0,2361^{\dagger}$ \\
$\quad$ Xifoide & $3 \pm 2$ & $7 \pm 4$ & $0,0113^{\dagger}$ \\
$\quad$ Umbilical & $2 \pm 1$ & $6 \pm 4$ & $0,0108^{\dagger}$ \\
\hline
\end{tabular}

Fonte: Dados da pesquisa.

Legenda: Os valores apresentados estão expressos em média \pm DP. CVF = Capacidade Vital Forçada; $V_{E F}=$ Volume Ex-

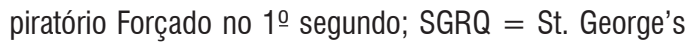
Respiratory Questionnaire; TC6min = distância percorrida no Teste de Caminhada de 6 Minutos; ${ }^{*}=$ Valor de $p$ do teste $t$ de Student pareado; ${ }^{\dagger}=$ Valor de $p$ do teste de Wilcoxon para amostras pareadas. estatisticamente significativa na distância percorrida determinada pelo TC6min e na Cirtometria nos níveis xifoide e umbilical quando comparados início e final do tratamento.

Após a aplicação do programa de exercícios, verificou-se um aumento significativo na mobilidade da região inferior da caixa torácica (cirtometria da região xifoide: de $3 \pm 2 \mathrm{~cm}$ para $7 \pm 4 \mathrm{~cm}, \mathrm{p}=0,01$ ) e na região abdominal (cirtometria umbilical: de $2 \pm$ $1 \mathrm{~cm}$ para $6 \pm 4 \mathrm{~cm}, \mathrm{p}=0,01$ ).

Observou-se uma melhora estatisticamente significativa da capacidade de exercício por meio do desempenho no TC6min, aumentando a distância percorrida quando comparada ao início do programa (de $391 \pm 117 \mathrm{~m}$ para $442 \pm 124$ metros, $\mathrm{p}=0,0403$ ).

Em relação à qualidade de vida, avaliada pelo SGRQ, houve uma tendência de melhora nos três domínios (sintomas: $37 \pm 22$ para $26 \pm 21$; atividades: $64 \pm 15$ para $62 \pm 19$; e impacto: $42 \pm 16$ para 38 \pm 16 ), porém os valores não alcançaram significância estatística.

\section{Discussão}

Os exercícios direcionados ao aumento da mobilidade da caixa torácica produziram melhora da expansibilidade torácica, da qualidade de vida e da capacidade de exercício nos pacientes deste estudo, além de uma resposta subjetiva de melhora na dispneia relatada.

A execução de atividades aeróbicas é necessária a fim de permitir uma autonomia social e física, tornando o paciente mais independente e ativo. Programas de Reabilitação Pulmonar aumentam a capacidade funcional e reduzem a sensação de dispneia em pacientes com DPOC $(2,7,14)$.

0 tratamento fisioterapêutico dos pacientes com DPOC visa aumentar a capacidade de realizar as atividades da vida diária, fazendo uso de exercícios que possam aumentar a mobilidade da caixa torácica e a força dos músculos respiratórios, ajudando, consequentemente, a prevenir a ocorrência de infecções respiratórias. Segundo Pitta et al. (23), indivíduos que não caminhavam mais do que 30 minutos por dia não aderiram à atividade física diária mínima preconizada em sua pesquisa, o que caracterizou, a cada dia de inatividade por semana, um aumento no risco de morte.

Os exercícios direcionados ao aumento da mobilidade da caixa torácica melhoram a expansibilidade 
torácica, a qualidade de vida e a capacidade submáxima ao exercício, reduzindo a dispneia e os níveis de depressão (20).

0 aumento mais acentuado nas regiões xifoide e umbilical, também encontrado por Paulin et al. (20), sugere a melhora da excursão diafragmática, porém sem alteração dos volumes e capacidades pulmonares. 0 estudo de Kakizaki et al. (25) evidenciou que pacientes com DPOC apresentam aumento de mobilidade na região torácica apical quando utilizados exercícios de alongamento da musculatura respiratória acessória. Paulin et al. (10) concluíram que os exercícios podem gerar alterações significativas após 10 sessões de treinamento.

A capacidade de exercício, avaliada pelo TC6min, apresentou uma melhora estatisticamente significativa após as 20 sessões. Paulin et al. (10) relatam que com a cinesioterapia respiratória, alguns aspectos fisiológicos não sofrem alterações; contudo, são os parâmetros funcionais que irão determinar de fato o benefício real de um determinado tratamento. 0 TC6min mostrou ser adequado para pacientes com DPOC, além de ser um recurso aplicável, reprodutível, prático e simples de ser realizado (26). Paulin et al. (27) relatam que o TC6min seria mais indicado por ser de fácil realização, com um desgaste menor para os pacientes e de mais baixo custo. Hernandes et al. (28) evidenciaram uma melhora significativa no desempenho durante o TC6min e ganho de capacidade aeróbia e uma correlação positiva entre o tempo de caminhada por dia em indivíduos com DPOC.

Observou-se uma tendência da melhora na qualidade de vida avaliada pelo SGRQ (18). Alterações acima de $10 \%$ refletem mudanças na qualidade de vida (30), sendo assim, é possível afirmar que houve mudanças no domínio "sintomas" após a aplicação do protocolo de exercícios.

Vale ressaltar que esse estudo apresentou como limitação metodológica o fato de ter sido empregada uma amostra com número pequeno de participantes, além da ausência de um grupo controle. No entanto, todos os benefícios obtidos vêm agregar a estudos similares, colaborando para o aumento do nível de validade científica do programa de exercícios.

Os pacientes apresentavam alterações espirométricas características da DPOC e essa condição pulmonar permaneceu inalterada após a aplicação do protocolo, situação já comprovada em outros estudos que mostraram que os valores da espirometria não se alteram com os programas de reabilitação (31).

\section{Conclusão}

Exercícios respiratórios direcionados ao aumento da mobilidade da caixa torácica melhoram a expansibilidade torácica inferior e abdominal e a capacidade de exercício em pacientes com DPOC. Esses efeitos concordam com outros estudos e colaboram para a formação de evidência científica para a inclusão desses exercícios nos programas de tratamento físico dos pacientes.

\section{Referências}

1. Zanchet RC, Viegas CA, Lima T. A eficácia da reabilitação pulmonar na capacidade de exercício, força da musculatura inspiratória e qualidade de vida de portadores de doença pulmonar obstrutiva crônica. J Pneumol. 2005;31(2):118-24.

2. Nici L, Donner C, Wouters E, Zuwallack R, Ambrosino $\mathrm{N}$, Bourbeau J, et al. American Thoracic Society/European Respiratory Society statement on pulmonary rehabilitation. Am J Respir Crit Care Med. 2006;173(12): 1390-413.

3. Velloso M, Jardim JR. Funcionalidade do paciente com doença pulmonar obstrutiva crônica e técnicas de conservação de energia. J Pneumol. 2006;32(6): 580-86.

4. Godoy DV, Godoy RF, Becker B Jr, Vacarri PF, Michelli M, Teixeira PJ, et al. The effect of psychotherapy provided as part of a pulmonary rehabilitation program for the treatment of patients with chronic obstructive pulmonary disease. J Pneumol. 2005;31(6): 449-505.

5. Godoy RF, Teixeira PJZ, Becker B Jr, Michelli M, Godoy DV. Repercussões tardias de um programa de reabilitação pulmonar sobre os índices de ansiedade, depressão, qualidade de vida e desempenho físico em portadores de DPOC. J Pneumol. 2009;35(2): 129-36.

6. Global Initiative For Chronic Obstructive Lung Disease (GOLD). Global Strategy for the Diagnosis, Management and Prevention of Chronic Obstructive Pulmonary Disease. NHLBI/WHO workshop report [text on the Internet]. Bethesda: National Heart, Lung and Blood Institute; 2001. [cited 2005 Jan 6]. Available at: http://www.goldcopd.com.2003. 
7. Dourado VZ, Antunes LCO, Tanni SE, Godoy I. Fatores associados à diferença clinicamente significativa da qualidade de vida relacionada à saúde após condicionamento físico em pacientes com DPOC. J Pneumol. 2009;35(9):846-53.

8. Gea J, Orozco-Levi M, Barreiro E. Particularidades fisiopatológicas de las alteraciones musculares en el paciente con EPOC. Nutr Hosp. 2006;21 Supl 3: 62-9.

9. Iturri JBG. Función de los músculos respiratorios en la EPOC. Arch Bronconeumol. 2000;36(5):275-85.

10. Paulin E, Tomio TC, Bueno GR, Barbosa VD, Oliveira EC, Riback NF. Efeito da cinesioterapia respiratória na mobilidade da caixa torácica, capacidade de exercício e qualidade de vida dos pacientes portadores de DPOC. Arq Cienc Saúde UNIPAR. 2006;10(3): 133-7.

11. Cooper CB, The connection between chronic obstructive pulmonary disease symptoms and hiperinflation and its impact on exercise and function. Am J Med. 2006;119(10 Suppl 1):21-31.

12. Godoy I. Avaliando a gravidade e o prognóstico da doença pulmonar obstrutiva crônica: a medida do $\mathrm{VEF}_{1}$ ainda é suficiente? J Pneumol. 2007;33(4): xxiii-xiv.

13. Mattos WLLD, Signori LGH, Borges FK, Bergamin JA, Machado V. Acurácia do exame clínico no diagnóstico da DPOC. J Pneumol. 2009;35(5):404-8.

14. Zanchet RC, Viegas CAA, Lima T. A eficácia da reabilitação pulmonar na capacidade de exercício, força da musculatura inspiratória e qualidade de vida de portadores de doença pulmonar obstrutiva crônica. J Pneumol. 2005;31(2):118-24.

15. Paulin E. Efeitos de um programa de exercícios físicos direcionado à mobilidade torácica na capacidade funcional e psicossocial em pacientes portadores de DPOC. [dissertação]. São Paulo: Universidade de São Paulo; 2002.

16. Sociedade Brasileira de Pneumologia e Tisiologia. Diretrizes para testes de função pulmonar. J Pneumol. 2002;28(3):S1-S82.

17. ATS statement: guidelines for the six-minute walk test. Am J Respir Crit Care Med 2002; 166(1):111117.
18. Camelier A, Rosa FW, Salmi C, Nascimento AO, Cardoso F, Jardim JR. Avaliação da qualidade de vida pelo questionário do Hospital Saint George na doença respiratória em portadores doença pulmonar obstrutiva crônica: validação de uma nova versão para o Brasil. J Pneumol. 2006;32(2):114-22.

19. Pitta F, Probst VS, Langer D, Trooters T, Gosselink R. Guia prático sobre o tratamento fisioterápico em pacientes com doença pulmonar obstrutiva crônica (DPOC): unindo evidências científicas e prática clínica. Rev Bras Fisioter. 2009;13(3):v-vi.

20. Paulin E, Brunetto AF, Carvalho CRF. Efeitos de programa de exercícios físicos direcionados ao aumento da mobilidade torácica em pacientes portadores de doença pulmonar obstrutiva crônica. J Pneumol. 2003; 29(5):287-94.

21. Puhan MA, Schünemann HJ, Frey M, Scharplatz M, Bachmann LM. How should COPD patients exercise during respiratory rehabilitation? Comparison of exercise modalities and intensities to treat skeletal muscle dysfunction. Thorax. 2005;60(5):367-75.

22. Silva EG, Dourado VZ. Treinamento de força para pacientes com doença pulmonar obstrutiva crônica. Rev Bras Med Esporte. 2008;14(3):231-8.

23. Pitta F, Troosters T, Probst VS, Lucas S, Decramer M, Gosselink R. Potential consequences for stable chronic obstructive pulmonary disease patients who do not get the recommended minimum daily amount of physical activity. J Pneumol. 2006;32(4):301-8.

24. Brunetto AF. Fisioterapia na DPOC: um sopro para a vida. Londrina: EDUEL; 2009.

25. Kakizaki F, Shibuya M, Yamazaki T, Yamada M, Suzuki H, Homma I. Preliminary report on the effects of respiratory muscle stretch gymnastics on chest wall mobility in patients with chronic obstructive pulmonary disease. Respir Care. 1999;44(4):409-14.

26. Pires SR, Oliveira AC, Parreira VF, Britto RR. Six-minute walk test at different ages and body mass indexes. Rev Bras Fisioter. 2007;11(2):131-4.

27. Paulin E, Brunetto AF, Alvares JL. Berticelli SM. Influência dos exercícios respiratórios direcionados à readequação do complexo toracopulmonar (RCTP) na saturação de oxigênio dos pacientes portadores de DPOC muito grave - Relato de casos. Arq Cienc Saúde UNIPAR. 2002;6(1):67-70. 
28. Hernandes NA, Teixeira DC, Probst VS, Brunetto AF, Ramos EMC, Pitta F. Perfil do nível de atividade física na vida diária de pacientes portadores de DPOC no Brasil. J Pneumol. 2009;35(10):949-56.

29. Sanchez FF, Faganello MM, Tanni SE, Lucheta PA, Padovani CR, Godoy I. Relationship between disease severity and quality of life in patients with chronic obstructive pulmonary disease. Braz J Med Biol Res. 2008;41(10):860-5.

30. Sousa TC, Jardim JR, Jones P. Validação do Questionário do Hospital de Saint George na Doença Respiratória (SGRQ) em pacientes portadores de doença pulmonar obstrutiva crônica no Brasil. J Pneumol. 2000;26(3):114-22.
31. Kunikoshita LN, Silva YP, Silva TLP, Costa D, Jamani M. Efeitos de três Programas de Fisioterapia Respiratória (PFR) em portadores de DPOC. Rev Bras Fisioter. 2006;10(4):449-55.

Recebido: 20/09/2011

Received: 09/20/2011

Aprovado: $25 / 03 / 2012$

Approved: 03/25/2012 